Trauma Berufskrankh 2014 • 16[Suppl 4]:366-371 DOI 10.1007/s10039-014-2113-3

Online publiziert: 10. Oktober 2014

c) Springer-Verlag Berlin Heidelberg 2014

\title{
T. Ideker
}

Deutsche Gesetzliche Unfallversicherung, Landesverband Nordwest, Hannover

\section{Neue Heilverfahren im Bereich der gesetzlichen Unfallversicherung} Aktueller Stand der Umsetzung

einerseits sowie der KBV (Kassenärztliche Bundesvereinigung) andererseits neu gefasst.

\section{H-Arzt-Verfahren}

\section{Beendigung}

Wesentliche Neuerung des Vertrags Ärzte/Unfallversicherungsträger ist die Beendigung des H-Arzt-Verfahrens (H-Arzt: an der Heilbehandlung beteiligter Arzt) zum 31.12.2015. Ab 01.01.2016 wird es in der ambulanten ärztlichen Versorgung Unfallverletzter nur noch das Durchgangsarztverfahren (D-Arzt-Verfahren) geben.

Für Ärzte, die am 01.01.2011 über eine Beteiligung am H-Arzt-Verfahren verfügten, besteht die Möglichkeit, auf Antrag in das D-Arzt-Verfahren, beschränkt auf eine ambulante Tätigkeit in der Niederlassung oder im Rahmen eines MVZ (Medizinisches Versorgungszentrum), zu wechseln. Der Antrag muss entsprechend der im Vertrag Ärzte/Unfallversicherungsträger getroffenen Vereinbarung bis zum 31.12.2014 beim zuständigen Landesverband der DGUV gestellt werden. Voraussetzungen für den erfolgreichen Wechsel sind u. a., dass die Praxis die baulichen und sächlichen Voraussetzungen nach den aktuellen D-Arzt-Anforderungen erfüllt. Auch muss die Mindestfallzahl von 250 erstversorgten Arbeitsunfallverletzten im Jahr erreicht werden. Maßstab hierfür bilden die übermittelten
H-Arzt-Berichte. Ein erneuter Nachweis der fachlichen Qualifikation ist dagegen nicht notwendig.

Zum 31.12.2010 waren bundesweit noch 2869 Ärzte am H-Arzt-Verfahren beteiligt, für die ab 01.01.2011 die Möglichkeit des Wechsels in das D-Arzt-Verfahren besteht. Bis zum 31.12.2013 sank die Anzahl gegenüber Ende 2010 um 630 auf 2239 H-Ärzte (-22\%). Im gleichen Zeitraum erhöhte sich jedoch die Anzahl der niedergelassenen Durchgangsärzte (DÄrzte), wozu auch die an einem MVZ tätigen D-Ärzte zählen, bundesweit nur um 301, von 2174 auf 2475 (+14\%, - Tab. 1).

\section{Inanspruchnahme des möglichen Wechsels in das Durchgangsarztverfahren}

Davon ausgehend, dass der Anstieg bei den niedergelassenen D-Ärzten nicht allein auf H-Ärzte zurückzuführen ist, die in das D-Arzt-Verfahren wechselten, sondern auch auf neu beteiligte D-Ärzte, machten bislang weniger als $10 \%$ der Ende 2010 beteiligten H-Ärzte von der Wechselmöglichkeit Gebrauch.

Die Ursachen hierfür sind vielfältig. $\mathrm{Zu}$ nennen sind $u$. a. hohe Investitions- und Vorhaltekosten, um die bisherige Praxis an die Anforderungen für das D-ArztVerfahren anzupassen, verknüpft mit einer zunehmenden Unsicherheit, besonders in ländlichen Regionen, die Praxis später einmal an einen Nachfolger übergeben zu können. Ein wesentlicher Grund 
Tab. 1 Veränderung der Anzahl der niedergelassenen D- und H-Ärzte zum Stichtag 31.12. der genannten Jahre ${ }^{a}$

\begin{tabular}{|lcccll}
\hline Bundesweit & 2010 & 2011 & 2013 & $\begin{array}{l}\text { Veränderung } \\
\text { gegenüber 2011 }\end{array}$ & $\begin{array}{l}\text { Veränderung } \\
\text { gegenüber 2010 }\end{array}$ \\
\hline D-Ärzte, niedergelassen & 2174 & 2297 & 2475 & +178 & +301 \\
\hline H-Ärzte & 2869 & 2685 & 2239 & -446 & -630 \\
\hline
\end{tabular}

D-Arzt Durchgangsarzt, DGUV Deutsche Gesetzliche Unfallversicherung, $H$-Arzt an der Heilbehandlung beteiligter Arzt

aStatistik der Landesverbände der DGUV

Tab. 2 Veränderungen im Bereich der Krankenhäuser mit Beteiligung am DAV, VAV oder SAV zum Stichtag 31.12. der genannten Jahre ${ }^{a}$

\begin{tabular}{lllll}
\hline Bundesweit & 2012 & 2013 & 2014 & Veränderung gegenüber 2012 \\
\hline DAV & 574 & 570 & 575 & \\
\hline VAV & 590 & 571 & 497 & \multirow{2}{*}{570} \\
\cline { 1 - 4 } SAV & - & - & 73 & \\
\hline Gesamt & 1164 & 1141 & 1145 & -19 \\
\hline
\end{tabular}

DAV Durchgangsarztverfahren, DGUV Deutsche Gesetzliche Unfallversicherung, SAV Schwerstverletzungsar-

tenverfahren, VAVVerletzungsartenverfahren

aStatistik der Landesverbände der DGUV

ist das Nichterreichen der notwendigen Mindestfallzahl von 250 erstversorgten Arbeitsunfallverletzten. Im Jahr 2012 lag die durchschnittliche Fallzahl im H-ArztVerfahren bundesweit nur bei 105 Fällen pro H-Arzt. Bei den niedergelassenen DÄrzten dagegen betrug die durchschnittliche Fallzahl 444 pro D-Arzt. Auch die Ausnahmeregelung für ländliche Regionen, wonach eine Unterschreitung der genannten Mindestfallzahl an erstversorgten Arbeitsunfallverletzten zulässig sein kann, wenn es ansonsten zu einer Gefährdung der Versorgung Arbeitsunfallverletzter in der Fläche kommen würde, konnten bislang nur wenige Antragsteller in Anspruch nehmen. Von einer Gefährdung der Versorgung Arbeitsunfallverletzter in der Fläche ist dann auszugehen, wenn sich das Verhältnis von Durchgangsärzten zu Versicherten in einem Landkreis oder einer kreisfreien Stadt schlechter als 1:30.000 darstellt oder in einer Region innerhalb von 30 min kein Durchgangsarzt zu erreichen ist [3]. Häufig aber sind ausreichend D-Ärzte in der näheren Umgebung zur Praxis des antragstellenden $\mathrm{H}$-Arztes vorhanden.

Aus diesen Gründen muss davon ausgegangen werden, dass es auch in den verbleibenden knapp 10 Monaten zu keinem deutlichen Anstieg von H-Ärzten kommen wird, die in das D-Arzt-Verfahren wechseln. Ab 01.01.2015 ist daher zu erwarten, dass der überwiegende An- teil der zurzeit noch beteiligten H-Ärzte an der ambulanten Versorgung Arbeitsunfallverletzter nicht mehr teilnehmen wird. Die bisherige Entwicklung bestätigt, dass das H-Arzt-Verfahren gegenüber dem D-Arzt-Verfahren in der ambulanten Versorgung Arbeitsunfallverletzter eine untergeordnete Rolle spielt. Die Beendigung dieses Verfahrens ist daher, insbesondere vor dem Hintergrund sinkender Unfallzahlen und unter dem Aspekt der Verbesserung von Qualität und Wirtschaftlichkeit, nur folgerichtig.

\section{Neue Anforderungen für das Durchgangsarztverfahren}

Die Beendigung des H-Arzt-Verfahrens zum 31.12.2015 führte zu einer wesentlichen Neuerung in den D-Arzt-Anforderungen ab 01.01.2011: Erstmals wurde eine Abstufung in der fachlichen Qualifikation vorgenommen. Weil eine Beteiligung am H-Arzt-Verfahren zuletzt überwiegend von Fachärzten für Orthopädie und Unfallchirurgie beantragt wurde, bildet diese Facharztqualifikation die Grundvoraussetzung zum Einstieg in die ambulante D-Arzt-Tätigkeit, auch Basis-D-Arzt genannt. Zusätzliche Voraussetzung, anknüpfend an den Facharzt für Orthopädie und Unfallchirurgie, ist der Nachweis einer mindestens 1-jährigen unfallchirurgischen Tätigkeit in einem am Verletzungsartenverfahren (VAV) beteilig- ten Krankenhaus, die in Vollzeit ausgeübt werden muss. Dadurch soll gewährleistet werden, dass Bewerber über ausreichende Kenntnisse und Erfahrungen in der Versorgung Unfallverletzter verfügen.

Der Basis-D-Arzt ist in der ambulanten Versorgung, d. h. in der Niederlassung oder in einem MVZ tätig, mit einem eingeschränkten Spektrum an ambulanten Eingriffen [4]. Wer dagegen ein weitergehendes Spektrum ambulanter Operationen [4] als D-Arzt anbieten oder als solcher an einem Krankenhaus stationäre Versorgungen erbringen möchte, muss weitergehende fachliche Qualifikationen nachweisen. Dafür sehen die D-Arzt-Anforderungen 2 Möglichkeiten vor:

- Facharzt für Chirurgie mit Schwerpunktbezeichnung Unfallchirurgie nach alter Weiterbildungsordnung oder

- Facharzt für Orthopädie und Unfallchirurgie mit der Zusatzqualifikation Spezielle Unfallchirurgie nach aktueller Weiterbildungsordnung.

Vor dem 01.01.2011 gab es in den D-ArztAnforderungen keine Differenzierung bei der fachlichen Qualifikation und im Umfang des Tätigkeitsspektrums. Der unterschiedlichen Versorgungsstruktur, besonders in ländlichen Regionen, wird auf diese Weise Rechnung getragen. Des Weiteren besteht nach Wegfall des H-Arzt-Verfahrens alternativ ein vereinfachter Einstieg in das D-Arzt-Verfahren für Fachärzte für Orthopädie und Unfallchirurgie.

\section{Stationäres Heilverfahren}

\section{Neuordnung ab 01.01.2013}

In einem 2. Schritt der Neuordnung der Heilverfahren in der GUV (Gesetzliche Unfallversicherung) traten zum 01.01.2013 die modifizierten bzw. neuen Verfahren für die stationäre Versorgung Arbeitsunfallverletzter in Kraft. Bis dahin gab es in diesem Bereich nur das Verletzungsartenverfahren (VAV) zur Versorgung SchwerUnfallverletzter. Die Zuordnung zu diesem Verfahren erfolgte über das sog. Verletzungsartenverzeichnis (VAV-Verzeichnis). Krankenhäuser mit einem D-Arzt, aber ohne Beteiligung am VAV, durften bislang stationäre Versorgungen von Ver- 
letzungen unterhalb des VAV-Verzeichnisses durchführen. Besondere strukturelle Anforderungen, die über die der DArzt-Anforderungen, die ausschließlich sächliche und bauliche Voraussetzungen für die Ambulanz oder Praxis beinhalteten, hinausgehen, gab es dafür nicht.

\section{Akutstationäre Versorgung}

In Anlehnung an das Weißbuch der DGU (Deutsche Gesellschaft für Unfallchirurgie, [1]) stehen nunmehr auch in der gesetzlichen Unfallversicherung ab 01.01.2013 für die akutstationäre Versorgung Arbeitsunfallverletzter 3 Verfahren zur Verfügung (• Abb. 1):

- Schwerstverletzungsartenverfahren (SAV),

- Verletzungsartenverfahren (VAV) und

- Durchgangsarztverfahren (DAV).

Oberhalb des VAV gibt es jetzt als höchste Versorgungsstufe zur Behandlung besonders schwerer und komplexer Verletzungen, die mit lang andauernden Arbeitsunfähigkeitszeiten, hoher Rentenrelevanz und hohem Rehabilitationsaufwand verbunden sind [5], das SAV. Die Zuordnung der Akutverletzten ins VAV oder SAV erfolgt weiterhin über das VAV-Verzeichnis in der ab 01.01.2013 modifizierten Fassung. Dieses ist wie bisher in 10 Verletzungsgruppen unterteilt. In den dazugehörigen Erläuterungen erfolgt eine weitergehende Differenzierung der einzelnen Gruppen. Die Verletzungen, die dem VAV zuzuordnen sind, sind nunmehr mit dem Zusatz (V), und die Verletzungen, die ihm Rahmen des SAV zu behandeln sind, mit dem Zusatz (S) gekennzeichnet. Unterhalb des VAV gibt es jetzt das stationäre Durchgangsarztverfahren (DAV), welches besondere Anforderungen für die akutstationäre Versorgung Unfallverletzter benennt, z. B. für die Notaufnahme oder die Operationsabteilung. Krankenhäuser mit D-Arzt, die diese Anforderungen zukünftig nicht erfüllen, dürfen akutstationäre Versorgungen von Arbeitsunfallverletzten nicht mehr durchführen. Dadurch sollen die Versorgungsqualität insgesamt gesteigert und die Krankenhäuser aufgewertet werden, die in relevanter Form Unfallchirurgie betreiben [5].

Trauma Berufskrankh 2014 · 16[Suppl 4]:366-371 DOI 10.1007/s10039-014-2113-3

(c) Springer-Verlag Berlin Heidelberg 2014

T. Ideker

Neue Heilverfahren im Bereich der gesetzlichen
Unfallversicherung. Aktueller Stand der Umsetzung

\section{Zusammenfassung}

Hintergrund. Die Neuordnung der Heilverfahren in der gesetzlichen Unfallversicherung wurde in 2 Schritten vollzogen.

Durchgangsarzt (D-Arzt)/H-Arzt. In einem ersten Schritt traten zum 01.01.2011 neue Anforderungen zur Beteiligung von Ärzten am Durchgangsarztverfahren in Kraft. Diese sehen erstmals eine Abstufung bei der fachlichen Qualifikation und im Umfang der Durchgangsarzttätigkeit vor. Gleichzeitig wurde mit der Kassenärztlichen Bundesvereinigung vereinbart, das bisherige $\mathrm{H}$-ArztVerfahren (H-Arzt: an der Heilbehandlung beteiligter Arzt) zum 31.12.2015 einzustellen. Vom 01.01.2016 an wird es im Bereich der ambulanten ärztlichen Versorgung Arbeitsunfallverletzter nur noch das Durchgangsarztverfahren geben.

Stationäre Heilverfahren. In einem zwei-

ten Schritt wurden zum 01.01.2013 die statio- nären Heilverfahren neu geordnet. Das bisherige Verletzungsartenverfahren (VAV) wurde modifiziert. Neu wurden das stationäre Durchgangsarztverfahren (DAV) und das Schwerstverletzungsartenverfahren (SAV) eingeführt. Letzteres konnte wie geplant zum 01.01.2014 mit bundesweit 73 Krankenhäusern starten. Eine weitgehend flächendeckende Versorgung Schwerstunfallverletzter ist sichergestellt. Viele der Kliniken erfüllen derzeit nicht in vollem Umfang die Anforderungen. Dies gilt auch für einige berufsgenossenschaftliche Unfallkrankenhäuser.

\section{Schlüsselwörter}

Unfallversicherung · Neuordnung ·

Arbeitsunfälle · Schwerst-Unfallverletzte .

Unfallkrankenhäuser

\section{New treatment procedures in the area of German Social Accident Insurance. Current state of implementation}

\section{Abstract}

Background. The restructuring of the course of patient treatment supported by the German Social Accident Insurance (DGUV) was executed in two steps.

Durchgangsarzt /H-doctors. On 1 January 2011 new requirements became effective regulating the participation of physicians in the Durchgangsarztverfahren-a special procedure regulating the treatment process for occupational accidents. The new requirements for the first time define a gradation of medical qualifications and the tasks of these specialized physicians. The DGUV and the Federation of Public Health Insurance-Contracted Doctors agreed to abandon the previous procedure involving so called $\mathrm{H}$-doctors by 31 December 2015. Effective 1 January 2016 there will only be the Durchgangsarztverfahren with regard to out-patient medical care for persons injured in an occupational accident.
In-patient medical treatment. In a second step the in-patient medical treatment was restructured. This measure went into effect on 1 January 2013. The existing VAV Injury Classification Procedure was modified and the treatment of the severely injured (SAV) and the in-patient treatment by specialized doctors (DAV) were introduced. SAV started as planned on 1 January 2014 with nationwide 73 certified hospitals, thus ensuring comprehensive medical care. However, some of the hospitals, including some BG clinics, currently do not fully meet the necessary requirements.

\section{Keywords}

Accident insurance $\cdot$ Restructuring . Accidents, occupational · Severely injured . Hospitalization

\section{Übergangsregelung bis 31.12.2017}

Mit Inkrafttreten der neuen stationären Heilverfahren ab 01.01.2013 wurde auch eine Übergangsregelung vereinbart.

Für das SAV besteht die Verlegungspflicht seit dem 01.01.2014. Das Jahr 2013 wurde von den Landesverbänden der DGUV benötigt, um Krankenhäuser auf Antrag an diesem Verfahren beteiligen zu können, nachdem die entsprechenden Voraussetzungen vorab vor Ort geprüft worden waren. 


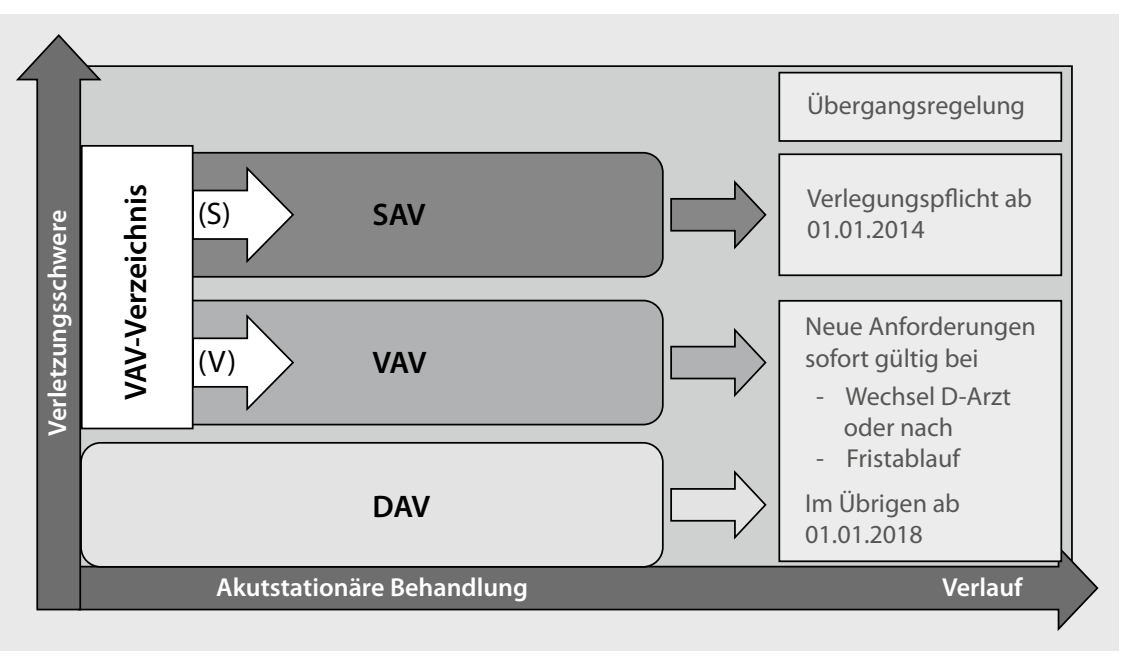

Abb. 1 \ Übersicht der neuen stationären Heilverfahren ab 01.01.2013 und der Übergangsregelung zur Anpassung an die neuen Verfahren, DAV Durchgangsarztverfahren, D-Arzt Durchgangsarzt, (S) Zusatz im Verletzungsartenverzeichnis für die Zuordnung zum SAV, SAV Schwerstverletzungsartenverfahren, (V) Zusatz im Verletzungsartenverzeichnis für die Zuordnung zum VAV, VAVVerletzungsartenverfahren

Die modifizierten Anforderungen für das VAV sind von den Krankenhäusern, die am 01.01.2013 an diesem Verfahren beteiligt waren, bis zum 31.12 .2017 umzusetzen oder aber sofort, sofern ein Wechsel des D-Arztes am Krankenhaus stattfindet oder eine bisher befristete Beteiligung an diesem Verfahren ausläuft. Ähnliches gilt für Krankenhäuser nur mit DArzt. Diese wurden zum 01.01.2013 in das DAV übernommen. Sie müssen spätestens zum 31.12.2017 die entsprechenden Anforderungen für das DAV erfüllen, bei einem Wechsel des D-Arztes allerdings sofort. Demzufolge werden spätestens ab 01.01.2018 nur noch Krankenhäuser am VAV oder DAV beteiligt sein, die die neuen Anforderungen erfüllen.

Ausgehend von den Bestandszahlen zum 31.12.2012 sind von diesem Veränderungsprozess der stationären Heilverfahren im DAV und VAV bundesweit 1164 Krankenhäuser betroffen, davon 574 Krankenhäuser mit D-Arzt (jetzt DAV) und 590 mit Beteiligung am VAV. Im Februar 2014 sind aktuell 575 Krankenhäuser im Rahmen der Übergangsregelung am DAV beteiligt und nur noch 497 am VAV. Der Rückgang von Krankenhäusern im VAV ist im Wesentlichen darauf zurückzuführen, dass 73 Krankenhäuser ab dem 01.01.2014 am SAV beteiligt sind. Im Gesamtbestand gab es gegenüber den Zahlen von Ende 2012 bundes- weit nur eine Veränderung von 19 Krankenhäusern. Dabei handelt es sich überwiegend um die Häuser, die nach Wechsel des D-Arztes die Anforderungen für das DAV nicht erfüllen konnten und damit an der akutstationären Versorgung Arbeitsunfallverletzter nicht mehr teilnehmen (- Tab. 2). Aufgrund der Übergangsregelung kam es im Bereich der am DAV und VAV beteiligten Krankenhäuser noch zu keinen wesentlichen Änderungen.

\section{Schwerstverletzungsartenverfahren}

\section{Sicherstellung der flächendeckenden Versorgung}

Ein besonderes Augenmerk liegt auf den ab 01.01.2014 bundesweit am SAV beteiligten 73 Krankenhäusern (• Abb. 2). Eine weitgehend flächendeckende Versorgung der Schwerst-Unfallverletzten im SAV kann dadurch sichergestellt werden. In einigen ländlichen Regionen, z. B. in Teilen von Mecklenburg-Vorpommern, Brandenburg, Niedersachsen oder auch in Bayern, sind die Entfernungen zu den SAV-Kliniken deutlich länger als in anderen Bundesländern. Im Notfall müssen in einem Krankenhaus, welches am DAV oder VAV beteiligt ist, zunächst eine Erstversorgung und Stabilisierung des Patienten erfolgen, um dann erst im zweiten Schritt, nach Eintritt von Transportfähigkeit, die Verlegung in das SAV-Kranken- haus durchführen zu können. Dies entspricht auch dem Grundgedanken eines Traumanetzwerks nach dem Weißbuch der DGU [1].

Auf das neue SAV entfallen nach erster Schätzung nur etwa 10-15\% der Fälle mit Verletzungen, die nach dem neuen VAVVerzeichnis mit (S) gekennzeichnet sind, d. h. die Mehrheit der Schwerverletzten kann wie bisher in den am VAV beteiligten Krankenhäusern versorgt werden.

Für den Landesverband Nordwest der DGUV mit den Bundesländern Schleswig-Holstein, Hamburg, Bremen, Niedersachsen und Sachsen-Anhalt sind, neben den 18 SAV-Krankenhäusern, auch die 70 am VAV beteiligten Kliniken dargestellt (• Abb. 3), die nach den Anforderungen und dem Weißbuch der DGU [1] zum TraumNetzwerk $\mathrm{DGU}^{\circ} \mathrm{zu}$ einer umfangreichen Erst- und, in Fällen des VAV, Definitivversorgung in der Lage sein müssen. Zur besseren Übersicht wurden die derzeit im Rahmen der Übergangsregelung am DAV beteiligten 105 Krankenhäuser nicht abgebildet. Diese kommen ebenfalls für eine ggf. notwendige Erstversorgung und Stabilisierung von Schwer-Unfallverletzten in Frage. Sofern vertretbar, sollen Schwerst-Unfallverletzte, also Fälle des SAV, durch den Rettungsdienst primär dem SAV-Krankenhaus zugeführt werden, um Sekundärtransporte zu vermeiden.

Wie die aktuelle Situation im Landesverband Nordwest der DGUV zeigt (- Abb. 3), ist die Versorgung von Schwer- und Schwerst-Unfallverletzten auch in ländlichen Regionen durch das SAV nicht gefährdet, da ausreichend Kliniken des VAV und DAV für eine ggf. notwendige ortsnahe Erstversorgung vorhanden sind.

\section{Beteiligungen überwiegend mit Auflagen}

Von den 18 Krankenhäusern, die im Bereich des Landesverbands Nordwest der DGUV ab 01.01.2014 am SAV beteiligt sind, erfolgte bei 17 die Beteiligung nur unter Auflagen. Diese betreffen im Wesentlichen bauliche und sächliche Anforderungskriterien, z. B.:

\section{Lage des Hubschrauberlandeplat-}

zes. Der Landeplatz muss in der Nä- 


\section{Neue Heilverfahren im Bereich der DGUV}

\begin{tabular}{|l|r|}
\hline \multicolumn{1}{|c|}{ Bundesland } & \multicolumn{1}{|c|}{ SAV-KH } \\
\hline Baden-Württemberg & 9 \\
\hline Bayern & 12 \\
\hline Berlin & 1 \\
\hline Brandenburg & 3 \\
\hline Bremen & 3 \\
\hline Hamburg & 6 \\
\hline Hessen & 2 \\
\hline Mecklenburg-Vorpommern & 7 \\
\hline Niedersachsen & 6 \\
\hline Nordrhein-Westfalen & 6 \\
\hline Rheinland-Pfalz & 2 \\
\hline Saarland & 5 \\
\hline Sachsen & 4 \\
\hline Sachsen-Anhalt & 3 \\
\hline Schleswig-Holstein & 3 \\
\hline Thüringen & 73 \\
\hline gesamt & \\
\hline
\end{tabular}

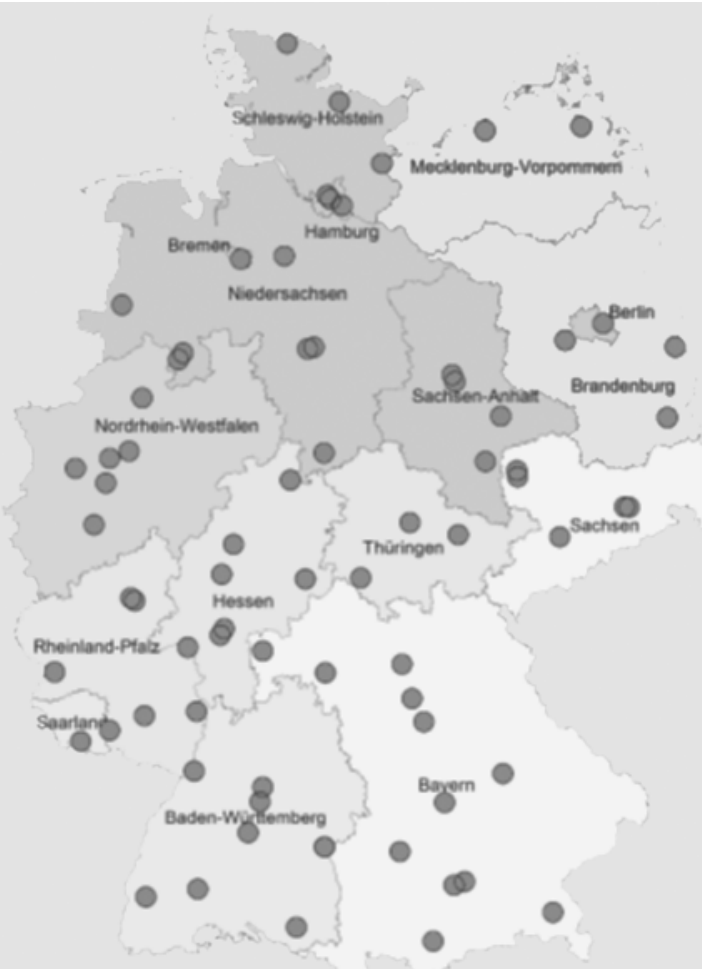

Abb. $2 \varangle$ Übersicht der bundesweiten SAV-Krankenhäuser, Stand Februar 2014, KH Krankenhaus, SAV Schwerstverletzungsartenverfahren. (Referat Statistik - Leistungen, Berufskrankheiten, Sonderaufgaben der DGUV)

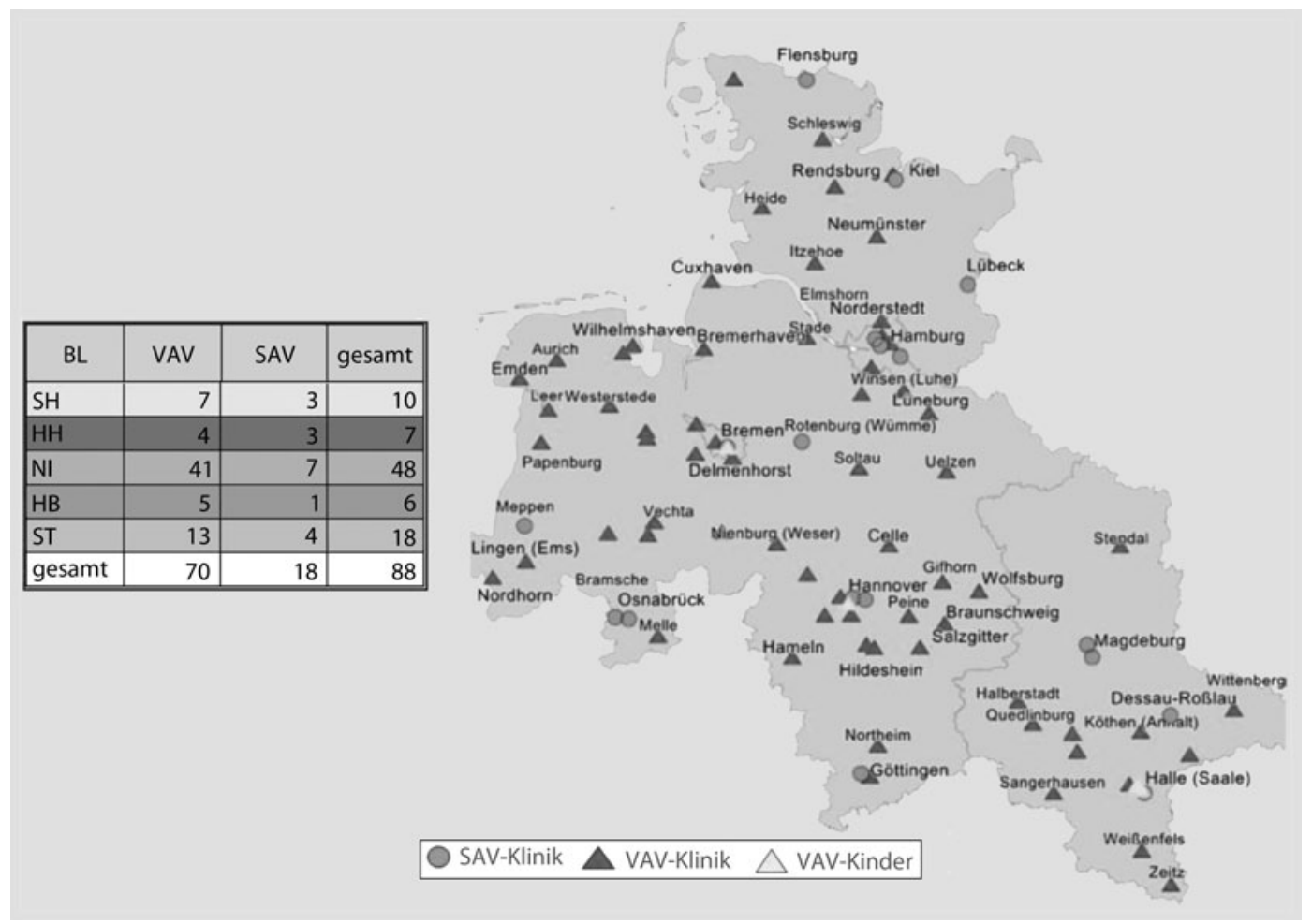

Abb. $3<$ Übersicht der SAV- und VAV-Krankenhäuser im Landesverband Nordwest der DGUV, Stand Februar 2014, BL Bundesland, DGUV Deutsche Gesetzliche Unfallversicherung, $H B$ Bremen, $H H$ Hamburg, NI Niedersachsen, SAV Schwerstverletzungsartenverfahren, SH Schleswig-Holstein, ST SachsenAnhalt, VAVVerletzungsartenverfahren. (Referat Statistik - Leistungen, Berufskrankheiten, Sonderaufgaben der DGUV)

he der Notaufnahme liegen, sodass der Transport des Patienten auf kurzem Weg, ohne zusätzliche Umlagerung in einen Rettungswagen, direkt in die Notaufnahme erfolgen kann.
Position des Computertomografen. Der Computertomograf muss sich in unmittelbarer Nähe zum Schockraum befinden.

Operationsabteilung. Sie muss baulich-funktionell und betrieblich-orga- nisatorisch den Empfehlungen des Robert Koch-Instituts (RKI) an die Hygiene bei Operationen und anderen invasive Eingriffen entsprechen. 
Psychotraumatologie. Eine ausreichende psychotraumatologische Kompetenz, u. a. zur frühzeitigen Identifikation und Therapie von posttraumatischen Belastungsstörungen, muss nachgewiesen sein.

Der hohe Anteil von Beteiligungen mit Auflagen zeigt, dass die Entwicklung im SAV noch am Anfang steht. Dies gilt auch für einige berufsgenossenschaftliche Unfallkrankenhäuser. Es werden weitere Krankenhäuser an dem Verfahren beteiligt werden, andere dagegen ihre Beteiligung wieder verlieren, weil Auflagen nicht oder nicht fristgerecht erfüllt werden können. Wie viele Krankenhäuser nach der Konsolidierungsphase am SAV bundesweit beteiligt sein werden, bleibt abzuwarten. Nach bisheriger Schätzung werden es voraussichtlich mehr als die zurzeit 73 Häuser sein.

\section{Resümee und Ausblick}

Im Bereich der ambulanten Versorgung Arbeitsunfallverletzter wird es ab dem 01.01.2016 nur noch das Durchgangsarztverfahren geben.

Bislang wechselten erst wenige H-Ärzte in das ambulante Durchgangsarztverfahren. Es muss davon ausgegangen werden, dass sich dies in den noch verbleibenden Monaten nicht gravierend ändern wird. Die meisten der jetzigen $\mathrm{H}$ Ärzte werden ab 01.01.2016 folglich nicht mehr an der ambulanten Versorgung Arbeitsunfallverletzter teilnehmen, womit sich die Anzahl der daran beteiligten Ärzte insgesamt ab diesem Zeitpunkt deutlich reduzieren wird. Die Anzahl der durchschnittlichen Fallzahlen der H-Ärzte im Vergleich zu denen niedergelassener D-Ärzte zeigt, dass das H-Arzt-Verfahren in der ambulanten Versorgung Arbeitsunfallverletzter nur eine untergeordnete Rolle spielt.

Erst nach Ablauf des Übergangszeitraums zum 31.12.2017 werden nur noch die Krankenhäuser am VAV und DAV beteiligt sein, die besonders erfahren und qualifiziert in der akutstationären Versorgung Unfall- und insbesondere SchwerUnfallverletzter sind und dafür die notwendigen strukturellen und personellen Voraussetzungen nach den neuen Anforderungen vorhalten. Die Anzahl der aktuell 1072 bundesweit am VAV und DAV be- teiligten Krankenhäuser (• Abb. 3) wird sich voraussichtlich weiter reduzieren, um wie viele Häuser, ist noch nicht absehbar.

Am SAV werden voraussichtlich mehr als die zurzeit 73 Krankenhäuser beteiligt sein. In einigen ländlichen Regionen kann es zu längeren Wegen für die Betroffenen kommen, aber nach derzeitiger Einschätzung zu keiner Unterversorgung im akutstationären Bereich.

Der dargestellte Entwicklungsstand zu den neuen Heilverfahren in der gesetzlichen Unfallversicherung zeigt, dass es in Zukunft bei der ambulanten und akutstationären Behandlung Arbeitsunfallverletzter zu einer weiteren Konzentration auf entsprechend beteiligte Ärzte und Krankenhäuser kommen wird, um die angestrebten Ziele des Eckpunktepapiers der DGUV, u. a. eine Verbesserung von Qualität und Wirtschaftlichkeit bei der Versorgung von schweren und schwersten Unfallverletzungen, erreichen zu können.

\section{Korrespondenzadresse}

\section{T. Ideker}

Deutsche Gesetzliche Unfallversicherung, Landesverband Nordwest, Hildesheimer Straße 309, 30519 Hannover Thomas.Ideker@dguv.de

\section{Einhaltung ethischer Richtlinien}

Interessenkonflikt. Th. Ideker gibt an, dass kein Interessenkonflikt besteht.

Dieser Beitrag beinhaltet keine Studien an Menschen oder Tieren.

The supplement containing this article is not sponsored by industry.

\section{Literatur}

1. Deutsche Gesellschaft für Unfallchirurgie e. V (2012) Weißbuch Schwerverletztenversorgung, Supplement 1, 2. erweiterte Aufl. DGU, Berlin, $\mathrm{S} 13 \mathrm{ff}$

2. DGUV (2009) Eckpunkte zur Neuausrichtung der Heilverfahren in der gesetzlichen Unfallversicherung. Rundschreiben DGUV - RS 0496/2009, vom 27.08.2009. DGUV, Berlin

3. DGUV, Spitzenverband der landwirtschaftlichen Sozialversicherung (2010) Auslegungsgrundsätze zu den Anforderungen der gesetzlichen Unfallversicherungsträger zur Beteiligung am Durchgangsarztverfahren in der Fassung vom 01.10.2010. DGUV, Berlin
4. DGUV, Spitzenverband der landwirtschaftlichen Sozialversicherung (2011) Grundsätze Ambulantes Operieren in der gesetzlichen Unfallversicherung, in der Fassung vom 1. Januar 2011. DGUV, Berlin

5. Oberscheven M (2013) Neuausrichtung der stationären Heilverfahren. Trauma Berufskrankh [Suppl 1] 15:25-28 